\title{
Pathogenesis and classification of massive periretinal proliferation
}

\author{
ROBERT MACHEMER \\ From the Bascom Palmer Eye Institute, Department of Ophthalmology, University of Miami School of \\ Medicine, Miami, and the Veterans Administration Hospital, Miami, Florida, USA
}

SUMMARY Massive periretinal proliferation (MPP), a serious complication of retinal detachment, is caused by proliferation and fibrous metaplasia of cells mostly deriving from retinal pigment epithelium and retinal glial cells. Contracting fibrous membranes in the vitreous, and on and also under the retina, cause the intraocular changes of MPP. Early signs such as increased 'tobacco dust', pigmented and unpigmented clumps in the vitreous, and subtle preretinal and even retroretinal membranes are usually overlooked. The late signs such as starfolds, irregular retinal folds, circumferential folds, and funnel-shaped detachments are well known. The pathogenesis of the clinically visible signs is described, and a 4-stage classification of the disease is given.

One of the most serious complications of retinal detachment is the development of excessive vitreous strand formation and fixed retinal folding. In accordance with its pathogenetic interpretation, this entity has been called massive vitreous retraction (MVR), massive preretinal retraction (MPR) (Tolentino et al., 1967), massive preretinal fibroplasia (Cibis, 1965), or massive periretinal poliferation (MPP) (Machemer and Laqua, 1975). This complication accounts for the majority of failures in retinal surgery.

Evaluation of animal experiments (Machemer and Laqua, 1975; Mandelcorn et al., 1975; MuellerJensen et al., 1975; Laqua and Machemer, 1975a; Laqua and Machemer, 1975b) in which the human disease could be simulated, the analysis of the intraocular situation during vitreous surgery, and the study of specimens taken from the human eye have all helped us to understand the human disease better.

In this paper the author's experiences are combined to explain the pathogenesis of the disease in the human eye and to present a clinically useful classification of its various stages.

\section{Pathogenesis of MPP}

Experimental production of retinal detachment in owl monkey eyes (Machemer and Norton, 1968)

Address for reprints: Dr Robert Machemer, Duke University Eye Center, PO Box 3802, Durham, North Carolina 27710, USA has provided a unique opportunity to study in detail the development of massive periretinal proliferation (MPP). The clinical picture of MPP in this model is very similar in appearance to that in the human disease. Histological examination of human eyes with MPP and electron microscopic studies of specimens obtained from human eyes during vitrectomy also suggest the similarity of the animal model to the human disease (van Horn et al., 1977).

Here an attempt is made to use the detailed knowledge gained from animal experiments and human studies to provide an explanation of the pathogenesis of MPP which is also valid for the human eye. Conclusions are based on the observations that cells undergoing proliferation and metaplasia probably deriving from retinal pigment epithelium (Machemer and Laqua, 1975; Mandelcorn et al., 1975; Mueller-Jensen et al., 1975; Machemer et al., 1978) and from retinal glial cells (Laqua and Machemer, 1975a; van Horn et al., 1977) are mostly responsible for this complication. The evidence for these two sources will be discussed in this order.

PIGMENT EPITHELIAL PROLIFERATION When the retina is detached, cells are probably liberated from the pigment epithelium and appear as pigmented macrophages under the retina (Machemer and Laqua, 1975). Like normal pigmented epithelial cells in contact with the outer surface of the retina these cells continue to phagocytose outer segment material (Fig. 1). They multiply 


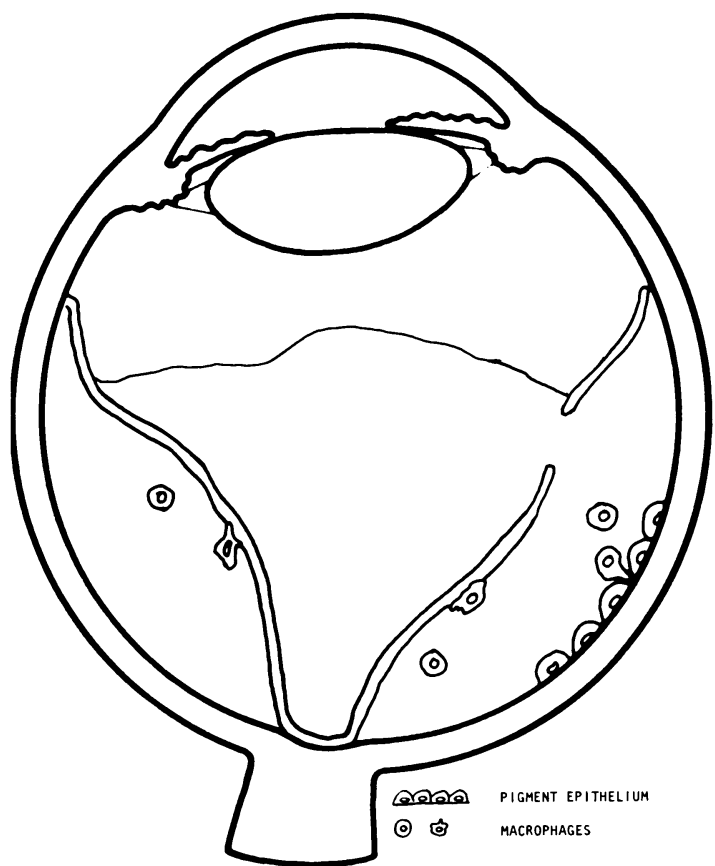

Fig. 1 Schematic drawing of rhegmatogenous retinal detachment. Pigment epithelial cells from the pigment epithelial layer are liberated and become macrophages either freely floating in subretinal fluid or phagocytosing outer segment material

and appear in the subretinal space as well as in the vitreous cavity (Fig. 2). Since the cells are not seen to migrate through the retina, they must reach the vitreous cavity through a retinal hole (Machemer and Laqua, 1975). The cells are usually visible as 'tobacco dust' (Hamilton and Taylor, 1972) in the vitreous and accumulate inferiorly on the surface of the retina.

Simultaneously some of the pigment epithelial macrophages undergo changes. A first group of cells begins to resemble fibroblasts and fibrocytes (Machemer and Laqua, 1975; Machemer et al., 1978). As the fibroblast-like cells multiply, clusters and tissue membranes of these cells are found on any available surface-the inner retinal surface, vitreal surface, posterior lens surface, and even in the subretinal space along the outer surface of the retina (Machemer and Laqua, 1975; Laqua and Machemer, 1975b) (Fig. 3).

A second group of cells also develop cellular membranes. Except for their ectopic location they bear close similarity to normal pigment epithelium and are therefore called pigment epithelium cells (Machemer and Laqua, 1975). Both types of cellular membranes are initially clinically invisible and can only be suspected by their slight pigmentation. Since the cells all originate from cells that have undergone many cell divisions, and pigment is not renewed during this process, their pigment content is very low (Machemer and Laqua, 1975) (Fig. 3).

\section{GLIAL CELL PROLIFERATION}

In addition to the pigment epithelial cells retinal glial cells participate in the proliferative process during retinal detachment (Laqua and Machemer, 1975a; van Horn et al., 1977). These cells grow through the internal limiting membrane and proliferate along the inner retinal surface, usually forming more localised membranes. Glial cells also protrude through the external limiting membrane of the retina and grow along its outer surface, sometimes covering large areas (Laqua and Machemer, 1975a) (Fig. 4).

CONTRACTION OF MEMBRANES

One of the most disastrous effects of massive periretinal proliferation is the sudden occurrence of contraction. A mechanism very similar to fibroblastic proliferation and contraction in wound repair (Peacock and Van Winkle, 1970) probably takes place inside the eye. Changed pigment epithelial

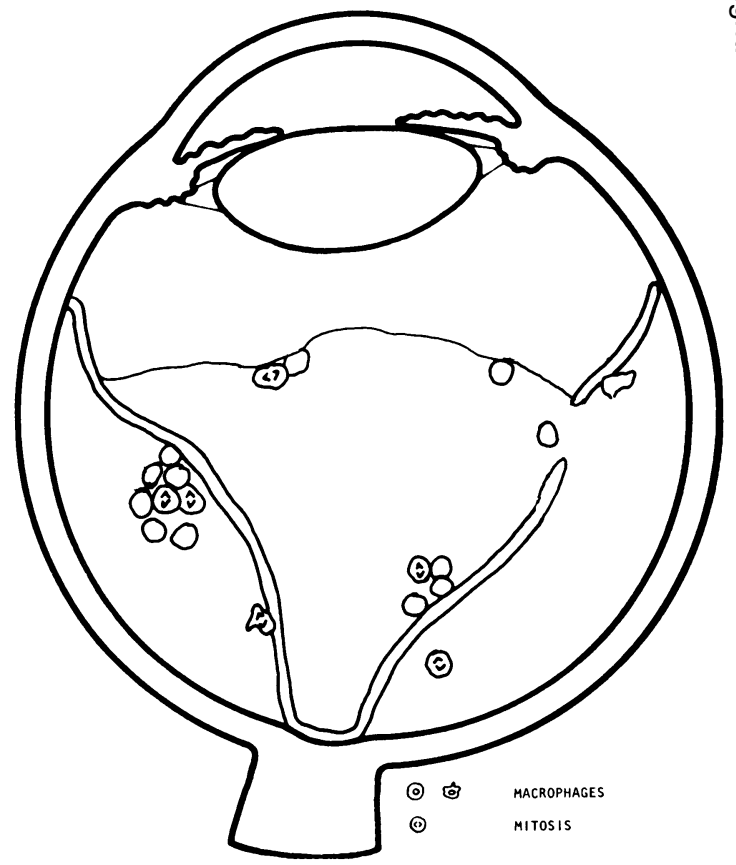

Fig. 2 Macrophages have migrated through the retinal hole into the vitreous cavity and settled on the retinal surface or posterior vitreous membrane. Their number increases through cell division 
cells behave like fibroblasts; they multiply and migrate in the direction of cell-free space along available surfaces. They adhere to the underlying surfaces and to each other. As the cells move forward they are stretched, and tensile or contractile forces develop (Constable et al., 1974).

Rather massive amounts of cytoplasmic filaments are found in the metaplastic pigment epithelium as well as in the proliferating glial cells (Machemer and Laqua, 1975; Laqua and Machemer, 1975a; van Horn et al., 1977; Machemer et al., 1978). Cytoplasmic filaments are a normal constituent of many cells. The exact importance of these filaments is not known, but it has been suggested that they may be contractile (Spoones et al., 1971; Crawford et al., 1972). The high content of these filaments in some of the membrane-forming cells makes it possible to speculate that it is this structure that causes contraction of the cells. Although cells are often not even interconnected by cell junctions, they seem to adhere to each other and form a membrane. It is this whole membrane that contracts.

Contractile forces are exerted very early in the development of the membranes. They are initially so minimal that changes remain invisible. The contractile forces finally become so strong that they

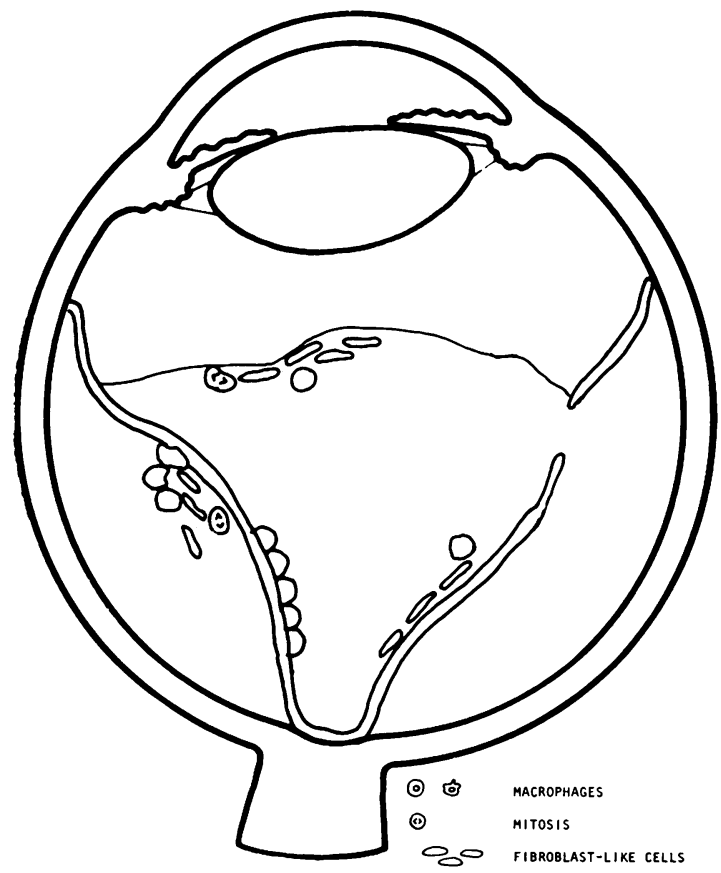

Fig. 3 Macrophages change into fibroblasts that form membranes under the retina, on the retinal surfaces, and along the detached posterior vitreous. Multiplication continues

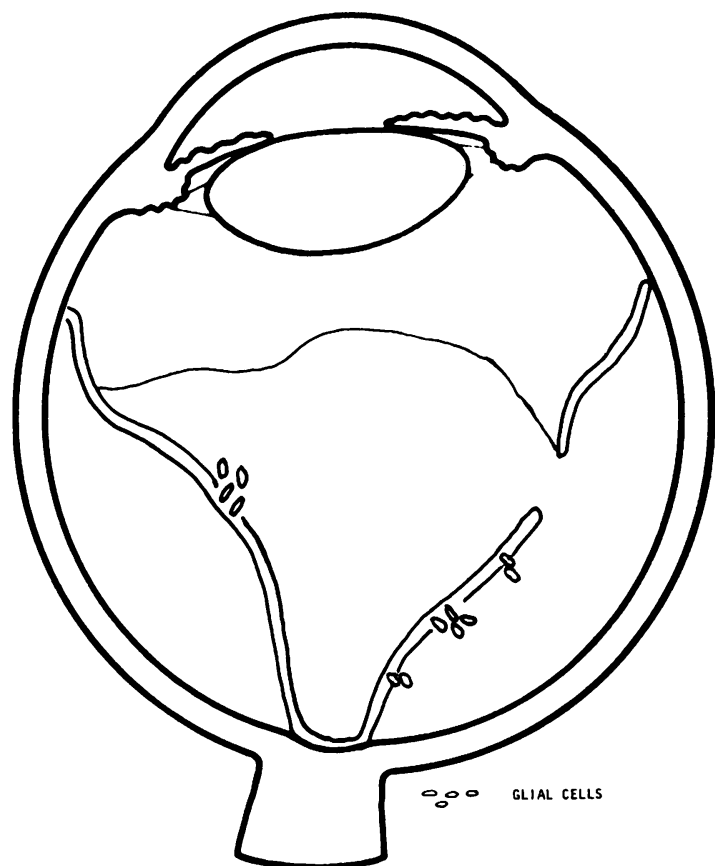

Fig. 4 Glial cells break through the internal and external limiting membrane of the retina and begin to form membranes on and under the retina

are stronger than the structural or adhesive forces of the retina. Depending on the force and the size of the contracting membrane, different clinical pictures result that vary from superficial surface wrinkling to heavy folding of the retina (Figs. 5, 6). Along the vitreous scaffolds contraction can occur without being seen, since vitreous structure itself is normally barely visible. If these membranes are on the surface of the retina, they can exert an extensive pull on the retina, resulting in its folding and detachment. Sometimes these membranes may be so large and the forces build up to such a degree that their contraction detaches the entire retina.

Experiments have shown that altered pigment epithelium can produce collagen (Machemer and Laqua, 1975; Mueller-Jensen et al., 1975; Newsome and Kenyon, 1973). Many workers have thought in the past, and most recently (Havener, 1973; Algvere and Kock, 1976) that this collagen formation is the key to the contraction of these membranes. However, it is known that collagen does not contract and exhibits shortening only when denatured (Peacock and Van Winkle, 1970). Therefore it must be concluded that the cells themselves produce the contractile forces. Collagen does not participate in the contraction but only in the stabilisation of the contracture (Peacock and Van Winkle, 1970). It is 


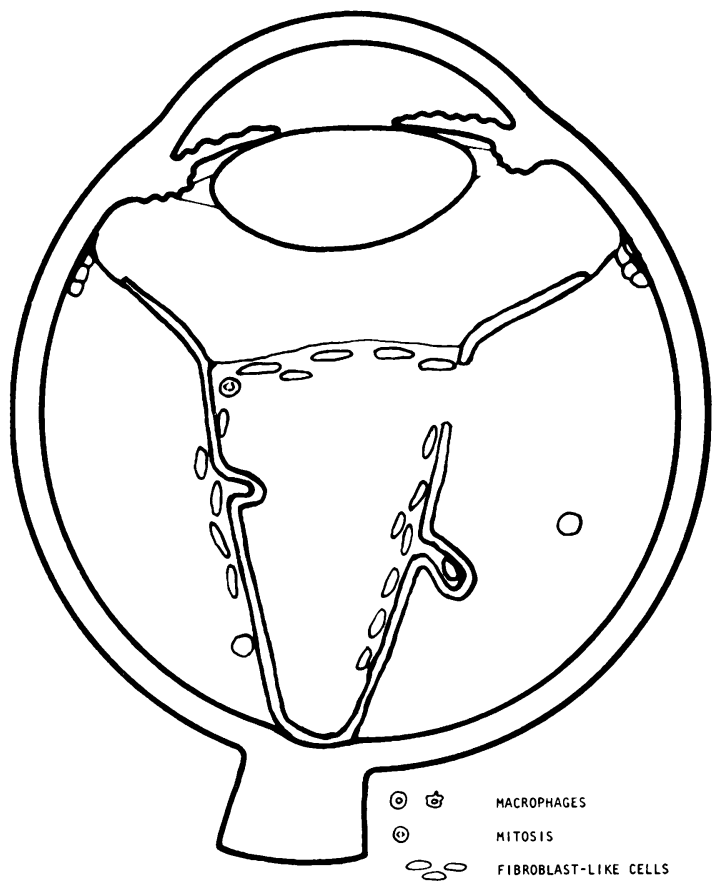

Fig. 5 Pre-and subretinal fibroblastic membranes contract and throw the retina into folds. Posterior vitreous membrane is shortened, pulling the retina centrally. A funnel-shaped detachment results

mainly the collagen component that makes the intravitreal tissue look whitish and forms the relatively solid intravitreal membranes (Fig. 7). Membranes are therefore not (or only to a very minor degree) condensations of pre-existing vitreous collagen. Preretinal membranes, which are practically invisible so long as they consist of only a simple cellular layer, lose their transparency after collagen deposition.

\section{Definition of MPP}

Massive periretinal proliferation (MPP) may thus be described as a condition of the eye with retinal detachment in which proliferation of cells along available surfaces-usually the vitreous, inner retinal, and outer retinal surfaces-results in membrane formation. The cells derive from pigment epithelium and retinal glia that have undergone metaplasia. The cellular membranes contract, causing the retina to fold and the vitreous framework to collapse. Deposition of collagen in the membranes renders them visible as semi-transparent intravitreal and preretinal strands and membranes.

Various pathogenetic mechanisms have been proposed in recent years to explain the development

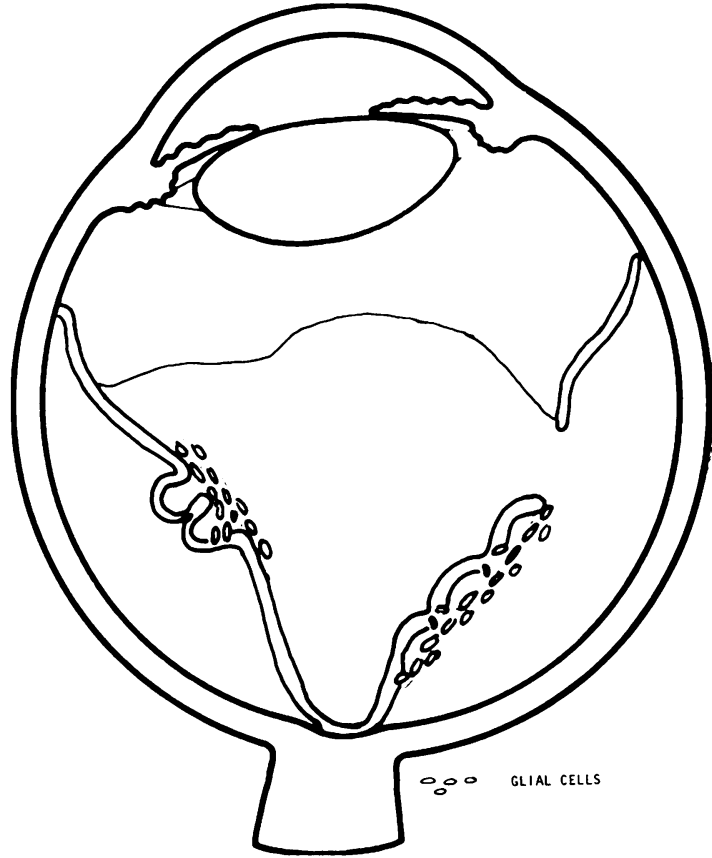

Fig. 6 Contracting pre- and subretinal glial membranes can cause retinal folding

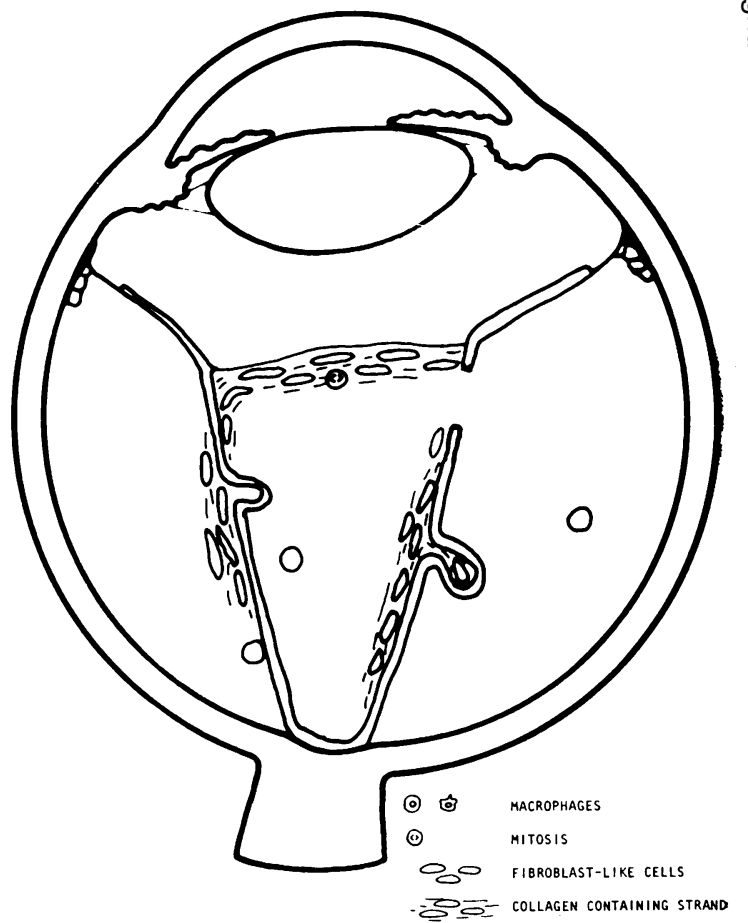

Fig. 7 Collagen deposition between fibrocytes stabilises contracted cellular membranes 


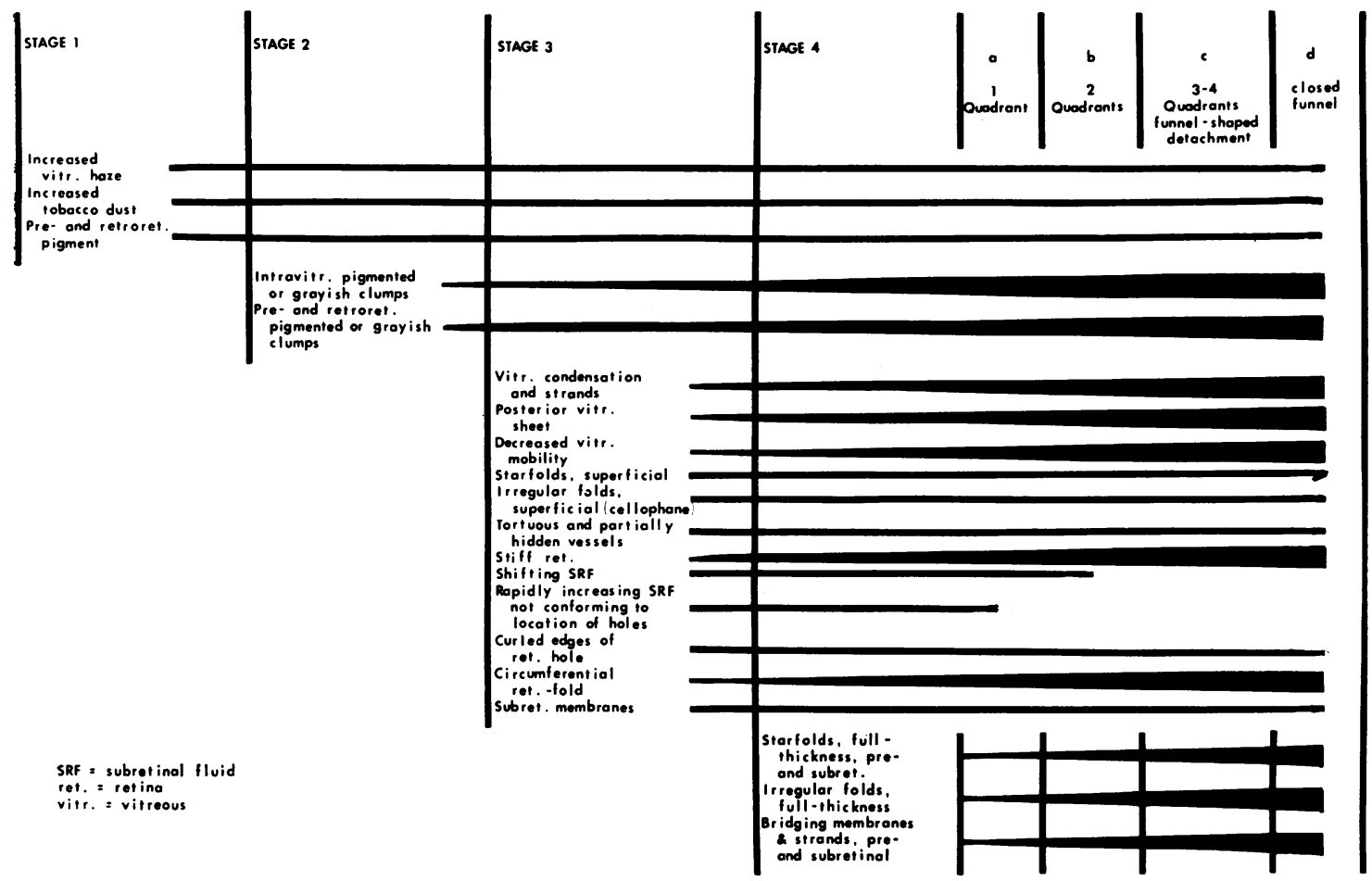

Fig. 8 Classification of massive periretinal proliferation (MPP). Continuous lines show presence of a symptom at the various stages. Expanding lines show increasing severity of a given symptom as the disease progresses

and clinical picture of massive periretinal proliferation. Just as the name implies, shrinkage of vitreous alone was originally thought to be the cause of massive vitreous retraction (MVR).

Havener (1973) in a rather recent detailed article describing MVR still adheres to this theory. Interestingly, he speculates that anterior vitreous separates from the vitreous base, which retracts posteriorly and forms a frontal plane. This idea is used to explain why the anterior retina usually looks smooth whereas the posterior retina is crinkled and why a plane crossing the vitreous cavity is seen. If biomicroscopy has not already shown that vitreous is condensed in the anterior vitreous cavity, vitrectomy certainly has proved that the vitreous in MVR is tightly adherent to the vitreous base and is detached posteriorly.

Tolentino et al. (1976) states that massive preretinal retraction (MPR) is caused by cells growing along the retinal surface, the posterior vitreous surface, and in the vitreous. They consider that shrinkage of the vitreous and of membranes (perhaps triggered by heat of diathermy and photocoagulation or cold from cryoapplications) causes the picture of MPR. The fibroblastic appearance of the membranes and their collagen content is emphasised (Smith,
1960; Constable et al., 1974). However, epithelial cells were seen as well.

Gloor (1976) agrees that MPR is caused by a proliferative process, but he thinks that it is caused by glial proliferation as suggested by the studies of Roth and Foos (1971), Foos (1974), Foos and Gloor (1975), and Rentsch (1973). He believes that tissue in the vitreous or along the posterior vitreous surface is actually a membrane that was 'ripped away' from the retina during vitreous detachment. Obviously, new proliferations of the retinal surface must occur to explain the folded retina behind such a posterior vitreous detachment. He also sees a continuum between macular fibrosis and massive preretinal retraction. I can agree with this observation only so long as spontaneous puckers are excluded. These are always localised and never end up in a massive shrinking process. In contrast, postoperative puckers after photocoagulation and buckling therapy of retinal holes can develop into a process involving the whole fundus. The localised nature of spontaneous puckers and the tendency of postoperative puckers occasionally to cause the full picture of MPP lets me assume that the latter are not solely caused by glial cells but rather by another type of cells, presumably cells deriving from the pigment epithelium. 


\section{Classification of MPP}

One of the main difficulties in the interpretation or comparison of results in the surgical treatment of MPP is the lack of a classification to define the various stages of its severity. Present classifications have rather broad stages and neglect the early signs. Tolentino et al. (1967), for example, differentiate between 'early' and 'established'; Havener (1973) between 'possible', 'probable', 'definite', and 'advanced'; Scott (1975) between 'prefibrotic' and 'fibrotic'. For this reason I consider it is important to redefine MPP stages more precisely and show these as detailed as possible in Fig. 8. In reading the descriptions of the various stages, it will again become obvious how the full picture of MPP develops.

STAGE 1

The vitreous, normally transparent, begins to have a diffuse haze. Isolated pigment epithelial macrophages appear as fine brown dots under the retina in the vitreous cavity and tend to accumulate on the surface of the inferior retina. Under the retina they usually remain undetectable unless a convoluted retinal flap allows observation of the undersurface of the retina (Laqua and Machemer, 1975b). In the vitreous cavity the pigmented macrophages are known as 'tobacco dust' (Hamilton and Taylor, 1972). These findings are not typical simply for MPP but are encountered in practically all rhegmatogenous retinal detachments. However, the vitreous haze and the number of brown dots markedly increase in MPP.

\section{STAGE 2}

Further proliferation of the macrophages results in cluster formation. These clusters become visible clinically as lightly pigmented or greyish clumps in the vitreous cavity and on the retinal surface and also as yellowish brownish clumps under the detached retina (Machemer and Laqua, 1975; Machemer and Norton, 1968). The intravitreal clumps have been described by Havener (1973), Scott (1976), and Tolentino et al. (1967). Whereas 'tobacco dust' (Hamilton and Taylor, 1972) represents only a large number of isolated macrophages (Machemer and Laqua, 1975), clump formation is the first indication of cells beginning to stick together and to form membranes (Machemer and Laqua, 1975; Laqua and Machemer, 1975b).

STAGE 3

Often the third stage of MPP is not fully appreciated clinically although it contains all the features that to a larger extent occur in full-blown MPP. The critical point is that cellular membranes are now developed along all the available surfaces.

The vitreous at this stage begins to show increased density and to develop a membrane along its detached posterior surface (Tolentino et al., 1967; Havener, 1973). Strands seen in the anterior vitreous may fuse with this membrane. Often the membrane has one or more round to oval openings or gaps, which often contain vitreous with an anteroposterior orientation of fibrils (Havener, 1973). The membranes are under tension and decrease the mobility of the vitreous.

Preretinal membranes are often not visible as such, though secondary changes are indicative of the membrane.

I have been able to verify in man the tiny superficial starfold which was seen in animal experiments (Laqua and Machemer, 1975b), which wrinkles only the surface of the retina in a very localised area (Fig. 9). This is possible only when the vitreous is clear enough to allow good visualisation of the retina and with the help of a contact lens and high magnification.

Large thin membranes on the surface of the retina cause irregular reflexes (cellophane appearance) because of wrinkling of the internal limiting membrane and irregularities on the membrane surface. Vessels become less visible. Slight contraction of the membranes results in tortuosity of the vessels (Laqua and Machemer, 1975b) (Fig. 10) or in their stretching outside the area of the membrane. The retina becomes stiff. Normal detached retina undulates easily after quick eye movements; the retina in MPP retains its position nearly unchanged. Together with this one observes a shifting of the subretinal fluid, which is an indication that the retina, though seemingly flattened, is no longer adherent to the pigment epithelium. The increasing preretinal membrane formation and the consequent

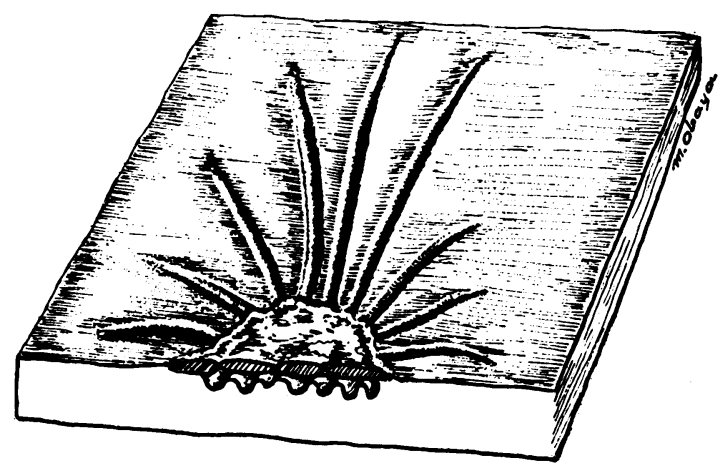

Fig. 9 Tiny superficial starfold created by small preretinal membrane folding superficial layers only (Permission American Journal of Ophthalmology) 


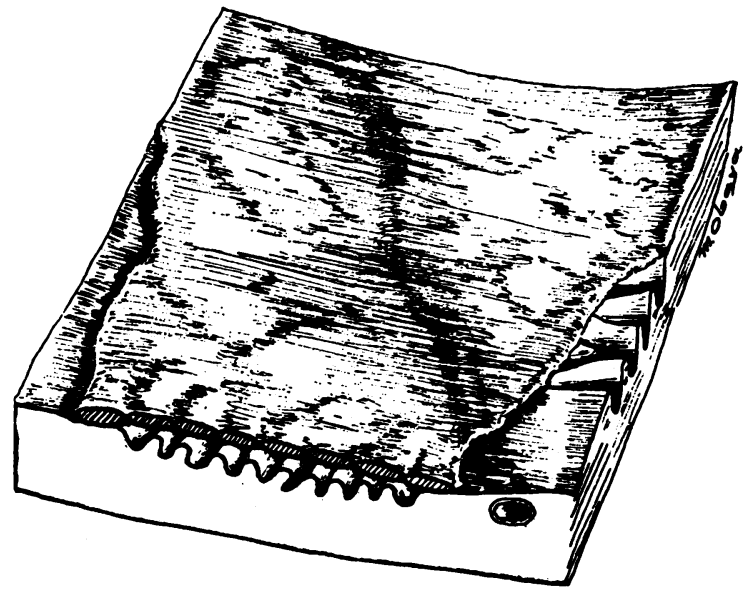

Fig. 10 Schematic drawing of preretinal membrane causing tortuosity and obscuration of vessels and also crinkling of internal retinal layer (Permission American Journal of Ophthalmology)

stiffening of the retina also account for the fact that the location of the subretinal fluid no longer conforms to the location of the retinal hole.

Impressive changes also occur at the edges of the retinal holes. Since there is relatively little resistance to contraction of these small preretinal membranes, the posterior edges of the holes tend to curl inwards. This sign has recently been emphasised by Scott (1976).

It is difficult to demonstrate thin subretinal membranous sheets when seen through an altered retina. However, they do exist and have in fact been peeled from the undersurface of a folded retina during vitreous surgery by the author.

\section{STAGE 4}

During the fourth stage membranes become more pronounced. They are contracted heavily and are visible because of increased collagen deposits.

The posterior vitreous membrane is very prominent and the anterior vitreous is heavily condensed and contains strands and pigmented cell clusters. With further contraction of the posterior vitreous membrane and with the retina being pulled increasingly towards the centre of the eye the space in the anterior vitreous cavity is reduced. Vitreous may be forced posteriorly through the gaps in the posterior vitreous membrane. This explains the anteroposterior orientation of the vitreous fibrils seen in these holes.

Contraction of localised preretinal membranes typically leads to the formation of isolated, fullthickness starfolds (Laqua and Machemer, 1975b) with a depressed centre and converging folds that stop just before the centre (Figs. 11a,b). Contraction of a localised subretinal membrane also causes a full-thickness starfold very similar in appearance to the better-known preretinal one (Laqua and Machemer, 1975b). Careful analysis of this fold reveals that the centre is raised instead of depressed and that the radiating folds are centripetally connected to each other instead of terminating before the centre (Figs. 12a, $b$ ).

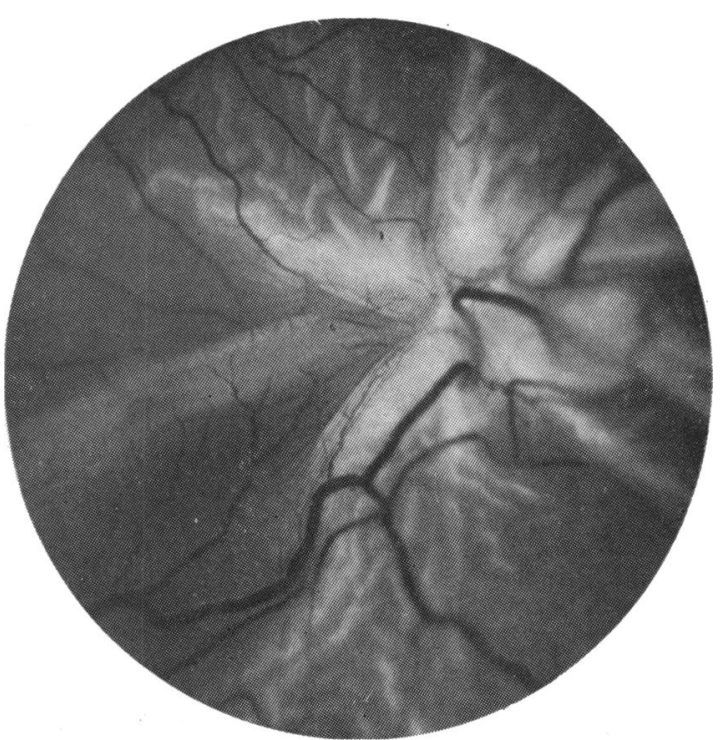

Fig. 11a Clinical photograph of full-thickness starfold caused by preretinal proliferation. Note the depressed centre and the converging folds stopping just before the centre

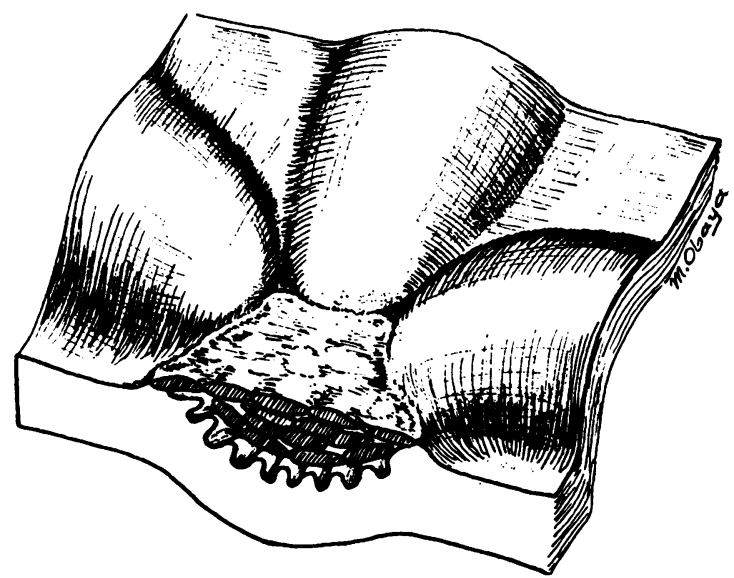

Fig. 11b Schematic drawing of full-thickness starfold caused by preretinal proliferation (Permission American Journal of Ophthalmology) 


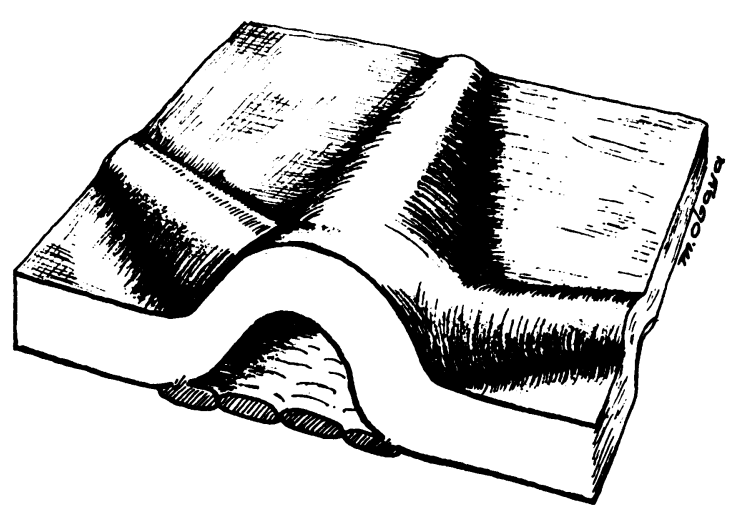

Fig. 12a Schematic drawing of full-thickness starfold caused by retroretinal proliferation. Note the raised centre and the folds that are in connection with each other (Permission American Journal of Ophthalmology)

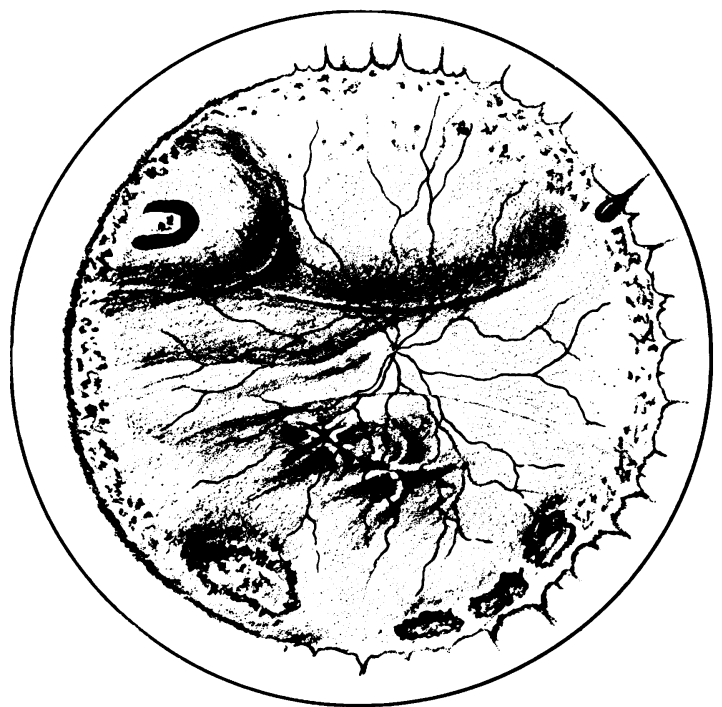

Fig. 12b Drawing of full-thickness starfolds with preretinal (left) and retroretinal proliferation (right) (taken with permission from Schepens (1960))

The retina is now irregularly folded; it looks thickened and has a yellow colour. These folds can be caused by a shrinking preretinal membrane (Fig. 13a) and are called full-thickness irregular folds. One is sometimes able to recognise strands bridging across the cascade-like retinal folds, but usually the folds are simply pulled tightly together. Vessels disappear completely in these folds (Fig. 13b). Subretinal folds-difficult to diagnose clinically but confirmed at vitreous surgery in folded-over retinacan also cause the full-thickness of the retina to fold (Fig. 14).

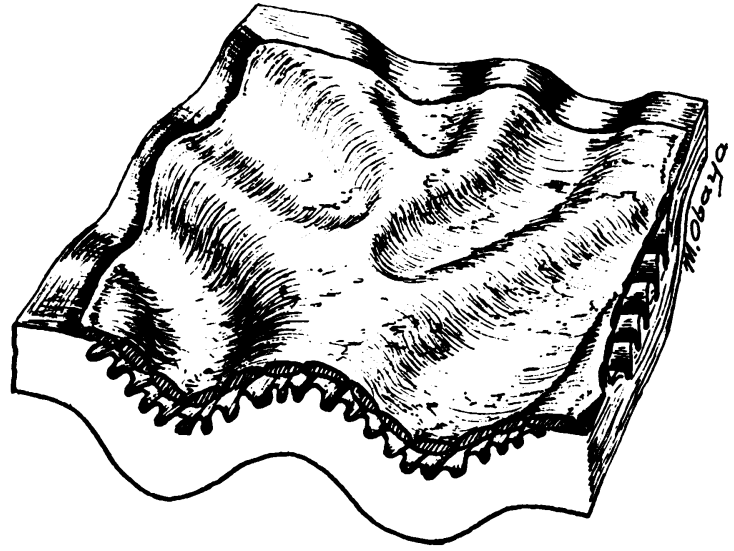

Fig. 13a Full-thickness irregular fold caused by large contracting preretinal membrane (Permission American Journal of Ophthalmology)

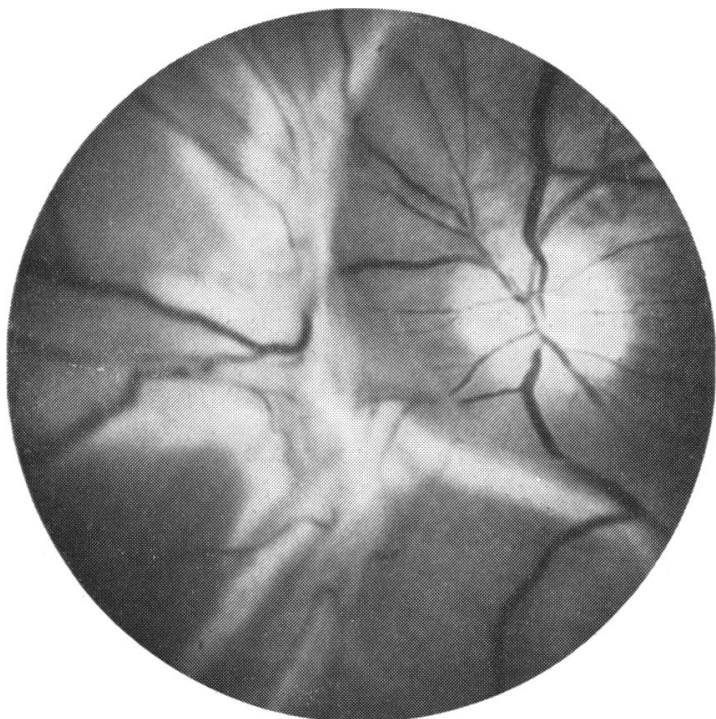

Fig. 13b Clinical photograph of full-thickness irregular fold. Tightly packed folds in which vessels disappear and bridging preretinal strands are visible

Very typical for this stage is the development of a circumferential retinal fold, which is usually located in the equatorial plane. First suggestions of this fold begin to show in stage 3 . The fold represents the border between the detached posterior vitreous surface and the adherent vitreous base, and often presents itself as an irregular, peripheral, greyish ring-like structure (Fig. 15). It is interesting to note that, while the retina posterior to the circumferential fold is usually irregularly folded, it is flat anteriorly except for some radial folds. This phenomenon can be understood when one remembers that cells 


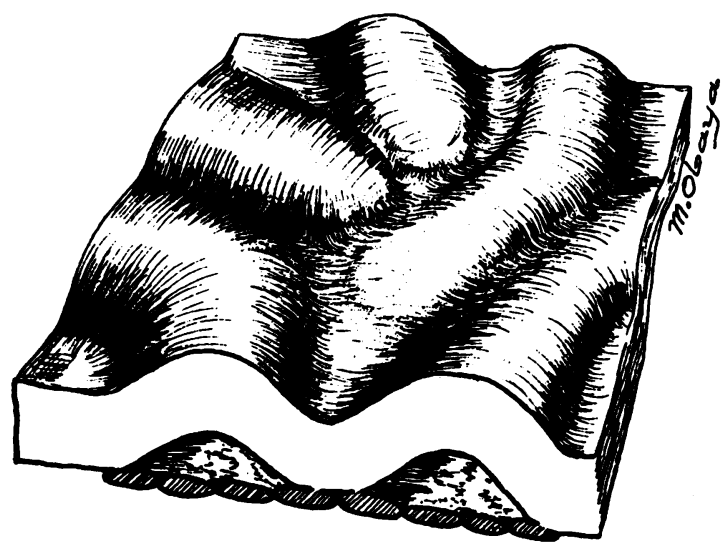

Fig. 14 Full-thickness irregular fold caused by subretinal membrane contraction (permission American Journal of Ophthalmology)

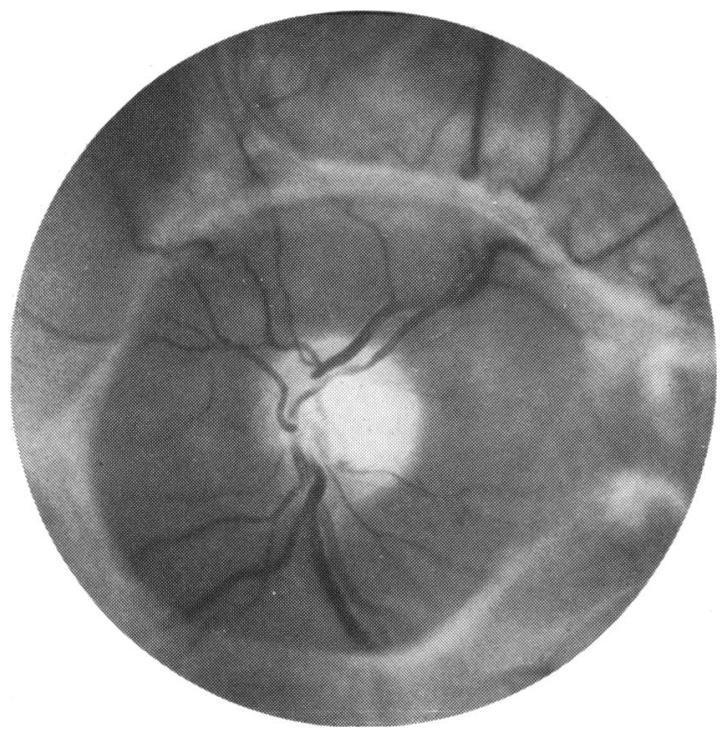

Fig. 15 Clinical photograph of circumferential retinal fold caused by a greyish ring of fibrous tissue. In this specific case the fold is well visible, since retina is not folded posteriorly

proliferate only along available surfaces. The vitreous base is still covered by vitreous and the proliferating cells do not have access to the peripheral retinal surface. In addition the contracting posterior vitreous membrane exerts a pull on the peripheral retina and stretches it (Fig. 16).

When all the posterior retinal surface is folded and the posterior vitreous plane has contracted, the typical funnel-shaped picture of MPP has developed, with the anterior retina in a frontal plane and the posterior retina forming a steep funnel (Fig. 17a). The contraction of the posterior vitreous surface can be so intense that eventually the disc itself may become invisible and the funnel entirely closed (Fig. 17b).

Because of the irregular surface of the retina and its decreased transparency it is very difficult to visualise anything that occurs underneath. But occasionally one will see definite strands after the retina has been reattached. These appear as straight, sometimes pigmented lines. These subretinal strands originally were part of a subretinal membrane.

This subretinal membrane does not adhere strongly to the retina (Laqua and Machemer, 1975a). When it begins to contract, it tears in those areas with the least thickness and the least tensile strength. Large holes develop in the membrane, and only the walls between the holes remain visible.

Finally, in the attempt to classify fully the changes of MPP for descriptive and therapeutic purposes I have found it necessary to subgroup this stage 4 . The subgroupings represent an ever increasing amount of pathology. Group $4 a$ represents a stage in which the lesion is confined to 1 quadrant; $4 b$ has involvement of 2 quadrants; $4 c$ involves 3 to 4 quadrants with a 'morning glory' appearance of the retina; in $4 d$ the funnel of the detached retina is so narrow that the disc becomes invisible. Groups $4 a$ and $b$ have been treated surgically with conventional buckling procedures and successes have been

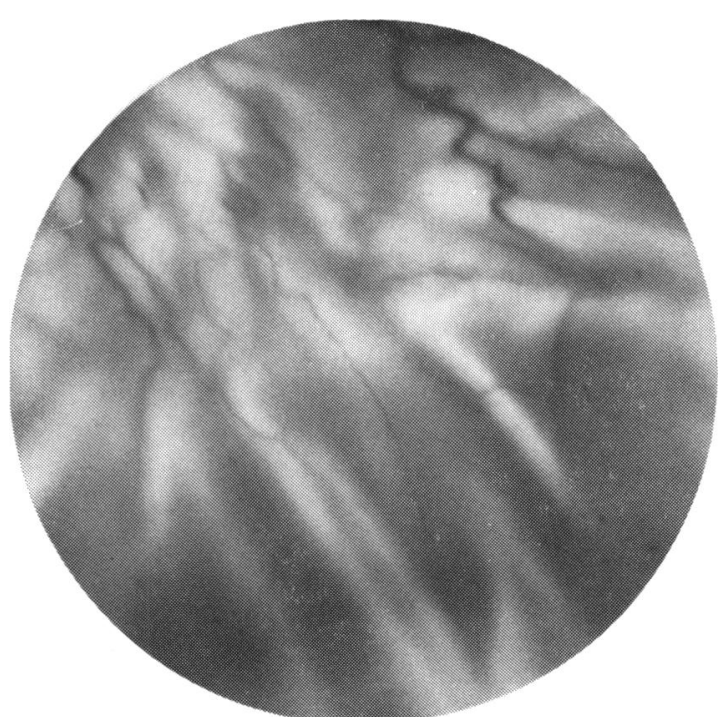

Fig. 16 Clinical photograph of peripheral retina in MPP. While the posterior retina is heavily folded, the peripheral retina appears stretched with some radial folds only.

In this case the fibrous ring is not visible 


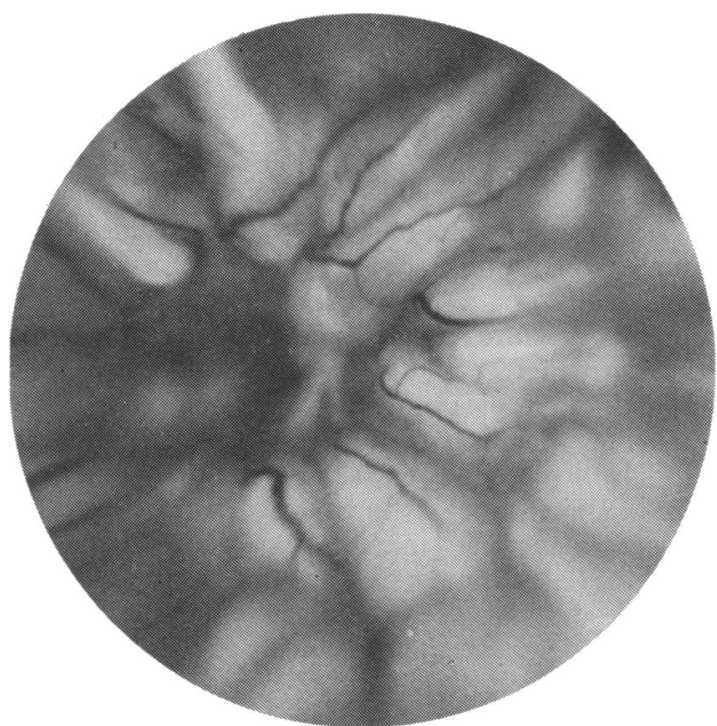

Fig. 17a Clinical photograph of central part of funnelshaped detachment with grade 4c MPP. Multiple cascade-like tightly packed full-thickness retinal folds in 3 quadrants pull the retina centrally. Note that the disc is still visible (out of focus)

achieved. Group $4 c$ constitutes the present domain for intravitreal silicone injections. No successes have been reported in Group 4d. A justification for vitreous surgery with removal of the vitreous and preretinal membrane peeling increases from Group $4 a$ to $4 d$.

\section{Conclusion}

MPP presents a tremendously wide range varying from proliferation of a few macrophages under a detached retina or on the retina and in the vitreous cavity to localised or, finally, generalised membrane formation and contraction of pigment epithelial and glial cells. This proliferation may become stationary or it may continue over many months at a low level, or it may be explosive, creating within hours the fullblown picture of MPP.

For the clinician it is of vital importance to find the earliest possible signs so as to have some idea of the impending disaster. I think that pigmented or greyish cell clusters in the vitreous cavity are probably the most important early clinical signs of active proliferation of cells. Together with increased vitreous haze they represent the first warning signs of possible impending complications. However, one must keep in mind that the proliferative process can stop at any stage during the development of MPP and that it still remains impossible to

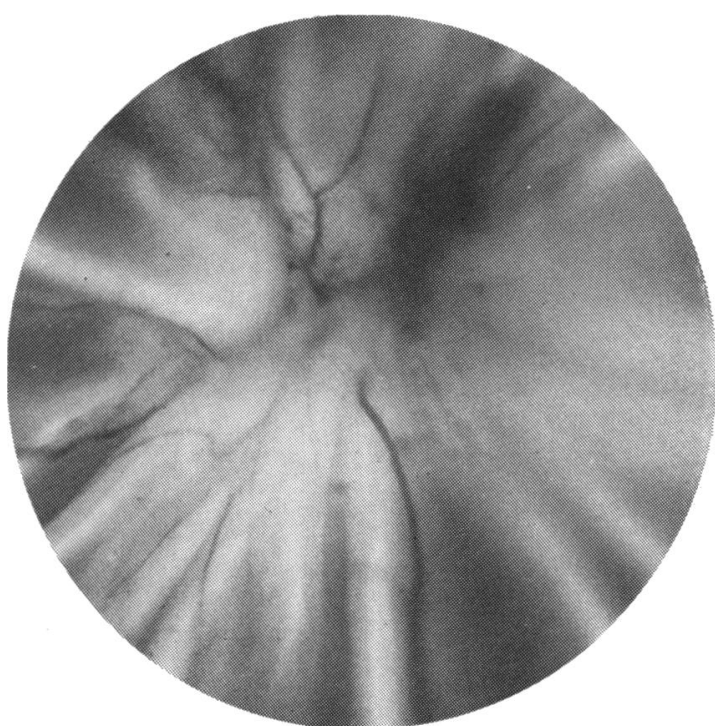

Fig. 17b Clinical photograph of retina with closed funnel (grade 4d MPP). Pigmented tissue pulls retina together so tightly that the disc is invisible. Peripheral retina is stretched and has only radial folds

predict in a given case that a specific lesion will develop further.

Horst Laqua, MD, Thomas M. Aaberg, MD, and Thorne Shipley, PhD, have critically reviewed this paper and helped with many suggestions.

This investigation was supported in part by NIH Research Grant EY-00841, the Veterans Administration Hospital, Miami, Florida, and the Florida Lions Eye Bank Inc., Miami, Florida 33152.

This paper is part of a thesis submitted in partial fulfilment of requirements for membership in the American Ophthalmological Society, May 1977.

\section{References}

Algvere, P., and Kock, E. (1976). Experimental fibroplasia in the rabbit vitreous. Retinal detachment induced by autologous fibroblasts. Albrecht von Graefes Archiv für klinische und experimentelle Ophthalomolgie, 199, 215-222.

Cibis, P. (1965). Vitreoretinal Pathology and Surgery in Retinal Detachment. Mosby: St. Louis.

Constable, I. J., Tolentino, F. I., Donovan, R. H., and Schepens, C. L. (1974). Clinical-pathologic correlation of vitreous membranes. In Retina Congress, pp. 245-257. Edited by R. C. Pruett and Ch. D. J. Regan. AppletonCentury-Crofts: New York.

Crawford, B., Cloney, R. A., and Cahn, R. D. (1972). Cloned pigmented retinal cells. The effects of cytochalasin on ultrastructure and behaviour. Zeitschrift für Zellforschung, 130, 135-152.

Foos, R. Y. (1974). Vitreoretinal juncture-simple epiretinal 
membranes. Albrecht von Graefes Archiv für klinische und experimentelle Ophthalomolgie, 189, 231.

Foos, R. Y., and Gloor, B. P. (1975). Vitreoretinal juncture; healing of experimental wounds. Albrecht von Graefes Archiv für klinische und experimentelle Ophthalomolgie, 196, 213.

Gloor, B. P. (1976). Macular fibrosis and massive preretinal retraction. Documenta Ophthalmologica Proceedings Series, New Developments in Ophthalmology, Vol. 7, p. 105. Edited by A. F. Deutman.

Hamilton, A. M., and Taylor, W. (1972). Significance of pigment granules in the vitreous. British Journal of Ophthalmology, 56, 700-702.

Havener, W. H. (1973). Massive vitreous retraction. Ophthalmic Surgery, 4, 22-67.

Laqua, H., and Machemer, R. (1975a). Glial cell proliferation in retinal detachment (Massive periretinal proliferation). American Journal of Ophthalmology, 80, 602-618.

Laqua, H., and Machemer, R. (1975b). Clinical-pathological correlation in massive periretinal proliferation. American Journal of Ophthalmology, 80, 913-929.

Machemer, R., and Laqua, H. (1975). Pigment epithelial proliferation in retinal detachment (Massive periretinal proliferation). American Journal of Ophthalmology, 80, 1-23.

Machemer, R., and Norton, E. W. D. (1968). Experimental retinal detachment in the owl monkey. I. Methods of production and clinical picture. American Journal of Ophthalmology, 66, 388-395.

Machemer, R., van Horn, D. L., and Aaberg, T. M. (1978). Pigment epithelial proliferation in human retinal detachment with massive periretinal proliferation. American Journal of Ophthalmology, 85, 181-191.

Mandelcorn, M., Machemer, R., Fineberg, E., and Hersch, S. B. (1975). Proliferation and metaplasia of intravitreal retinal pigment epithelial cell autotransplants. American Journal of Ophthalmology, 80, 227-237.

Mueller-Jensen, K., Machemer, R., and Azarnia, R. (1975). Autotransplantation of retinal pigment epithelium in intravitreal diffusion chambers. American Journal of Ophthalmology, 80, 530-537.
Newsome, D. A., and Kenyon, K. R. (1973). Collagen production in vitro by the retinal pigmented epithelium of the chick embryo. Developmental Biology, 32, 387-400.

Peacock, E. E., and Van Winkle, W. (1970). Surgery and Biology of Wound Repair, p. 49. Saunders: Philadelphia.

Rentsch, F. J. (1973). Preretinal proliferations of glial cells after mechanical injury of the rabbit retina. Albrecht von Graefes Archiv für klinische und experimentelle Ophthalomolgie, 188, 79.

Roth, A. M., and Foos, R. Y. (1971). Surface wrinkling retinopathy in eyes enucleated at autopsy. Transactions of the American Academy of Ophthalmology and Otolaryngology, 75, 1047.

Schepens, C. L. (1960). Importance of the Vitreous Body in Retina Surgery with Special Emphasis on Reoperation. Mosby: St. Louis.

Scott, J. D. (1975). The treatment of massive vitreous retraction by the separation of preretinal membranes using liquid silicone. Modern Problems in Ophthalmology, 15, 285-290.

Scott, J. D. (1976). Treatment of massive vitreous retraction. Transactions of the Ophthalmological Societies of the United Kingdom, 95, 429-432.

Smith, T. (1960). Pathologic findings after retina surgery. In Importance of the Vitreous Body in Retina Surgery with Special Emphasis on Reoperations, p. 61-93. Edited by C. L. Schepens. Mosby: St. Louis.

Spoones, B. S., Yamada, K. M., and Wessels, N. K. (1971). Microfilaments and cell locomotion. Journal of Cell Biology, 49, 595-613.

Tolentino, F. I., Schepens, C. L., and Freeman, H. M. (1967). Massive preretinal retraction. Archives of Ophthalmology, 78, 16-22.

Tolentino, F. I., Schepens, C. L., and Freeman, H. M. (1976). Vitreoretinal Disorders, Diagnosis and Management, $\mathrm{p}$ 479-489. Saunders: Philadelphia.

van Horn, D. L., Aaberg, T. M., Machemer, R., and Fenzl, R. (1977). Glial cell proliferation in human retinal detachment with MPP (Massive periretinal proliferation). American Journal of Ophthalmology, 84, 383-393. 